\title{
Patient-specific total hip arthroplasty is superior to conventional methods for Crowe III and IV adult developmental hip dysplasia: a randomized controlled trial
}

\author{
Chenggong Wang ${ }^{1,2}$, Yusheng $\mathrm{Li}^{1}$, Yihe $\mathrm{Hu}^{1}$, Hua Liu ${ }^{1}$, Long Wang ${ }^{1}$, Jie Xie ${ }^{1}$, Han Xiao ${ }^{2}$, Shilong Su${ }^{2}$, \\ Fawei Gao ${ }^{2}$, Da Zhong ${ }^{1,2} \wedge$ \\ ${ }^{1}$ Department of Orthopedics, Xiangya Hospital Central South University, Changsha, China; ${ }^{2}$ Digital Research Institute of Orthopedics, Xiangya \\ Hospital Central South University, Changsha, China \\ Contributions: (I) Conception and design: D Zhong, C Wang; (II) Administrative support: Y Hu, H Liu; (III) Provision of study materials or patients: \\ D Zhong, Y Hu, Y Li, L Wang, J Xie; (IV) Collection and assembly of data: H Xiao, S Su, F Gao; (V) Data analysis and interpretation: C Wang, H \\ Xiao; (VI) Manuscript writing: All authors; (VII) Final approval of manuscript: All authors. \\ Correspondence to: Prof. Da Zhong, MD. Department of Orthopedics, Xiangya Hospital Central South University, No. 87 Xiangya Road, Changsha \\ 410008, China. Email: dr_zhongda@126.com.
}

Background: Restoration of the acetabulum during total hip arthroplasty in adults with developmental dysplasia of the hip (DDH-THA) and resumption of hip function remain major challenges. Herein, a new patient-specific instrument (PSI) was developed that uses the superolateral rim of the acetabulum as a positioning marker to assist surgeons in adult DDH-THA.

Methods: From January 2017 to October 2018, 104 adult DDH patients were randomized to either the PSI group or conventional operation (CO) group, and further divided into eight subgroups by stratified random sampling using Crowe's classification. Complications, Harris hip scores (HHS), and X-ray results were recorded at 3 and 12 months after surgery.

Results: With the exception of anteversion in CO-Crowe II group patients, there was no difference in the accuracy of cup placement and orientation between the PSI and CO groups in Crowe I and II DDH patients. With the exception of percentage of acetabular cup coverage (PACC) and the qualification rate of Crowe IV PACC patients, among all Crowe III and IV DDH groups, all postoperative indexes of cup orientation and positioning exhibited significant differences between the PSI and CO groups; however, no significant differences were observed in Crowe I and II DDH patients.

Conclusions: Compared with conventional methods, the new PSI-assisted surgical method improved the accuracy of placement and orientation of the acetabulum and cup prosthesis, optimized the surgical process, reduced complications, and contributed to quicker recovery of hip function after surgery in adults with Crowe III and IV DDH-THA, but little difference was noted for those with Crowe I and II DDH.

Keywords: Patient-specific instrument (PSI); total hip arthroplasty (THA); developmental dysplasia of the hip (DDH); Crowe classification; acetabulum

Submitted Apr 22, 2020. Accepted for publication Oct 30, 2020.

doi: 10.21037/atm-20-3488

View this article at: http://dx.doi.org/10.21037/atm-20-3488

$\wedge$ ORCID: 0000-0001-5733-0754. 


\section{Introduction}

Total hip arthroplasty (THA) is usually the preferred treatment for pain and end-stage osteoarthritis, secondary to developmental dysplasia of the hip (DDH) in adult patients $(1,2)$. Accurate preoperative planning and precise execution of that planning during THA are crucial (3). Especially in DDH-THA, the size, orientation, and position of the cup are important considerations for the recovery of hip joint function. Inaccurate placement of the cup can lead to severe postoperative complications $(4,5)$. At present, there is a general consensus for the type of acetabulum required for reconstruction in DDH patients. Many studies agree that whenever possible, the acetabular prosthesis should be installed in the true acetabulum $(6,7)$. Some researchers have noted that the displacement criteria should be $3 \mathrm{~mm}$ superior and $5 \mathrm{~mm}$ medial to the hip centre of rotation (COR) (8). Lewinnek suggested that reasonable anteversion should be $5-25^{\circ}$ and reasonable inclination should be $30-50^{\circ}$ (9). However, the location and size of the true acetabulum is difficult to determine because DDH could be the result of various anatomical abnormalities (10). Therefore, conventional THA surgical methods, which rely on manual freehand techniques, are both inconsistent and unsatisfactory $(11,12)$.

Many research groups have previously attempted to solve this problem. Some have advocated that the acetabular orientation and position can be determined by fully understanding the pathomorphology of the acetabulum and Harris fossa of all Crowe types (5). However, there are disadvantages to these methods, such as a steep learning curve and possibly extensive surgical exposure and injury (13). Some new digital technologies assisted surgery, such as computer navigation and augmented reality (AR), may be able to solve the problem, but studies on such technologies mostly focus on assisting THA in patients with primary hip osteoarthritis or femoral head avascular necrosis, and the results of these studies suggest that ideal solutions still cannot be found. Computer navigation techniques can assist with locating the true acetabulum and installing the guide cup (14-16). However, such techniques are expensive and impractical at some institutions. Furthermore, some studies have reported no improvement in accuracy and no significant benefit between conventional methods and navigational technology $(17,18)$. Kollamaram et al. (19) designed an AR-HIP system to aid in determining the proper orientation during surgery; however, its clinical feasibility remains unknown (20).
Therefore, a new method that can facilitate determination of the accurate location of the true acetabulum and precise orientation of the cup in adult DDH patients is necessary. The development of rapid prototyping technology (RPT) can now facilitate reconstruction or printing of the pelvis and simulate computerized surgery (21). Such techniques can aid in determining optimal acetabular size, orientation, and position, and simulate optimal cup implantation $(21,22)$. Thus, patient-specific instrument (PSI), based on preoperative simulated surgical design, may be a solution to the clinical challenges faced in adult DDHTHA. Recent reports have shown that PSI facilitated by three-dimensional (3D) printing can be used for acetabular component installation (23-25); however, the effects of such application were not ideal.

To solve the clinical challenges associated with DDHTHA, our team evaluated relevant research and designed a PSI, based on 3D reconstruction and RPT. The PSI uses the superolateral rim of the acetabulum as a positioning marker to aid in the production of an artificial acetabulum in adult DDH-THA. Six years ago, the technology passed a large number of model trials, and four years ago, it was approved by the ethics committee and subjected to clinical studies at our hospital.

We selected qualified cases from the artificial joint surgery registration system of our hospital. We aimed to verify whether the new PSI can indeed accurately and rapidly facilitate preoperative planning and the creation of an ideal artificial acetabulum for adult patients with Crowe II/III DDH during THA. To our knowledge, no randomized controlled trial (RCT) on the effect of PSI in adult DDH-THA has been conducted thus far. In view of the accumulation of cases treated by our team over the years, we conducted an RCT. We randomly divided adult study participants who had undergone DDH-THA into the PSI group or conventional operation (CO) group, to compare the accuracy of placement of the acetabular and cup prosthesis, the surgical processes, surgical complications, and postoperative hip function between the two methods. We present the following article in accordance with the CONSORT reporting checklist (available at http://dx.doi. org/10.21037/atm-20-3488).

\section{Methods}

\section{Study design and setting}

This RCT was conducted according to Declaration of 
Helsinki principles (as revised in 2013) and was conducted at our hospital from January 2017 to December 2019 (Registration number: ChiCTR1900024076). The study was approved by the medical ethics committee of the Xiangya Hospital of Centre South University (No. 201611080). Informed consent was obtained from all individual participants included in the study. The surgical procedures were performed between January 2017 and October 2018, and follow-up was conducted from January 2017 to December 2019.

\section{Participants}

Patients with a clinically and radiographically verified diagnosis of DDH were eligible for participation. The inclusion criteria were: (I) diagnosed with DDH; (II) firsttime THA; (III) aged 18-85 years; (IV) no other surgery performed on the ipsilateral hip joint; (V) operation on one side alone. The exclusion criteria were: (I) refusal to participate; (II) existence of severe osteoarthritis or other serious systemic diseases; (III) pregnancy; (IV) inability to complete regular follow-up; (V) patients in whom the contralateral hip was dysfunctional or who required bilateral surgery; (VI) mental disability; (VII) other reasons.

\section{Surgical protocol}

In both the PSI group and CO group, the surgical procedure was performed via a direct posterior-lateral approach in a lateral decubitus position by two senior surgeons. After cutting the femoral head, the anterior joint capsule was released to enlarge the field on the acetabular side. The synovial membrane and acetabular labrum were then resected. Surgical procedures in the CO group were performed according to traditional methods. However, surgical procedures in the PSI group were facilitated by the PSI. It is worth noting that the femoral surgical procedures in both groups were still performed according to traditional methods. Preoperative planning was required before surgery for both groups, but the PSI groups required preoperative design of the PSI before surgery.

\section{Preoperative planning}

Reconstruction of the pelvis and simulation of prosthetic implantation computed tomography (CT) scanning data of the pelvis in patients was performed by exporting the data from a Philips scanner (Philips, Eindhoven, The Netherlands) with $0.7-\mathrm{mm}$ slice thickness, and saving it in a Digital Imaging and Communications in Medicine (DICOM) format. Briefly, 3D models of the pelvis were digitally reconstructed using E-3D V17.08 software (Huiqing, Ltd., Nanjing, China). We first determined the coronal plane, based on the relative position of the anterior superior iliac spines and the pubic tubercles. The pelvic position was then standardized with reference to the anterior pelvic plane (26). We mimicked the implantation of the cup in the true acetabulum, according to the anatomical characteristics of the patient's acetabulum. The $3 \mathrm{D}$, sagittal, coronal, and transverse views were used to determine the ideal position, based on the following criteria: first, the diameter of the cup was confined by the peripheral border of the true acetabulum, to achieve rim fit. Second, we determined the optimal anteversion and inclination of the cup by using computerized surgical simulation (27). Third, the percentage of acetabular cup coverage (PACC) was obtained by stereo measurements based on the $3 \mathrm{D}$ CT reconstruction data. We designed the coverage to be greater than $70 \%$, based on the methods of Ueno et al. (27). Fourth, we designed the COR. We were able to duplicate the size of the femoral head and COR on the affected side if the contralateral head was normal. We tried to simulate the normal anatomical position of the COR if the contralateral head was abnormal. Furthermore, we ensured that all horizontal distances (HD) were less than $5 \mathrm{~mm}$ and all vertical distances (VD) were less than $3 \mathrm{~mm}(8)$ (Figures 1-3). We repaired large bony defects of the acetabulum with structural bone grafts or tantalum augmentation.

\section{Preoperative design of the PSI}

Once we determined the ideal cup size, orientation, and position, we developed a PSI to replicate the position of implantation during surgery. Three types of instruments were used: the first type was used for Crowe I and II DDHTHA, and the other two types were used for Crowe III and Crowe IV. Each type consisted of two parts (Figures 1-3). (I) The fitting part, which consisted of part A and part B. We chose part of the superolateral rim of the acetabulum and analyzed the surface to design a fitting part that matched the unique bony landmarks. This part was used to install the PSI against the bone. (II) The ground acetabulum part, which had three ends. The first end, the connecting rod, was designed to connect with the fitting part A; the second end, the guide plate of the reamer, had an arc-shaped end matching the surface of the rim of the simulated cup, and was designed to help guide reaming size, and depth and 

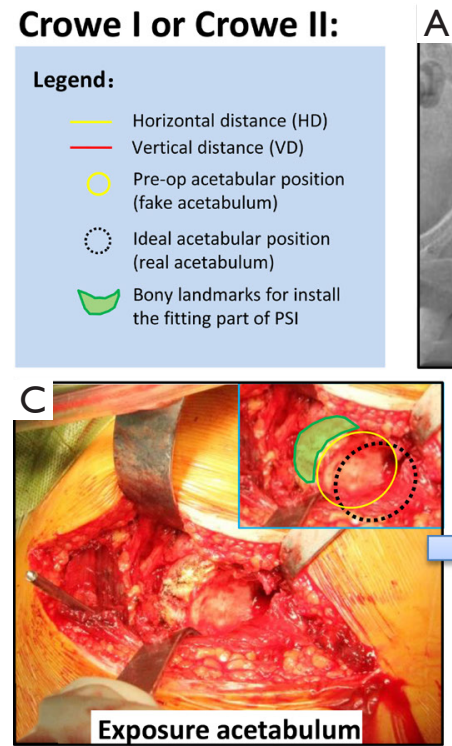
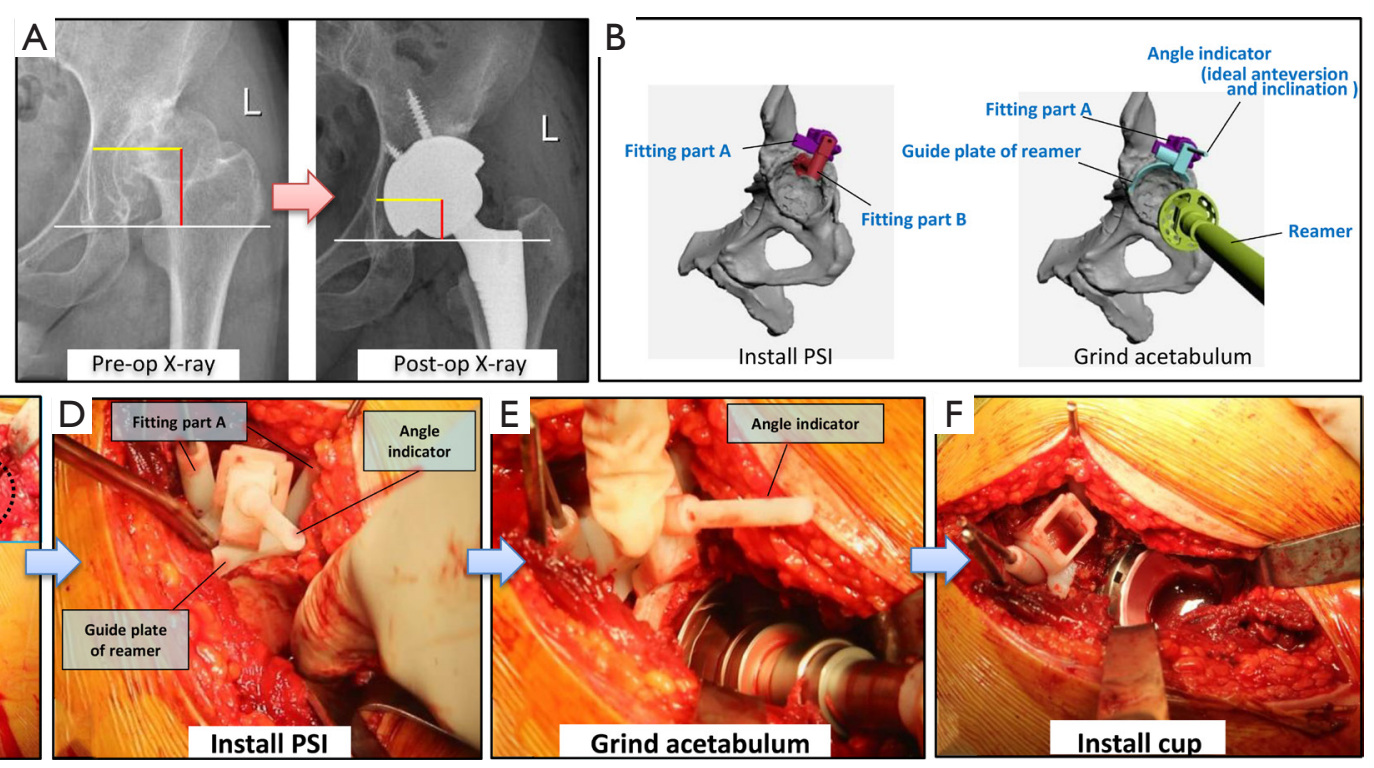

Figure 1 Details of application of the new patient-specific instrument (PSI) for patients with Crowe I or Crowe II developmental dysplasia of the hip (DDH). (A) Preoperative and postoperative pelvic radiographs of a typical case. The image shows that the centre of rotation (COR), horizontal distance (HD) and vertical distance (VD) have returned to normal after surgery. (B) Schematic diagram of the two main steps (installation of PSI and grinding down of the acetabulum) of the new PSI surgical method. (C,D,E,F) Application of the PSI during surgery.

\section{Crowe III:}
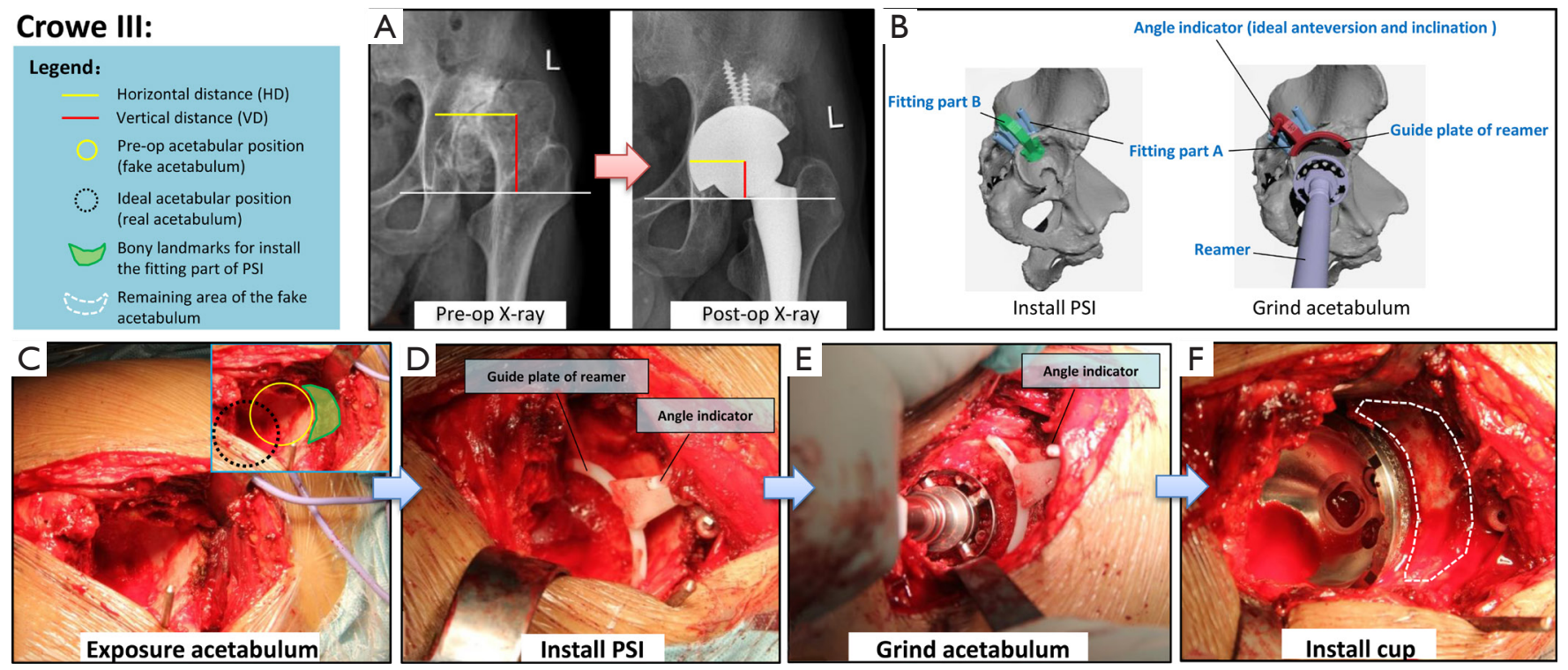

Figure 2 Details of application of the new patient-specific instrument (PSI) for patients with Crowe III developmental dysplasia of the hip (DDH). (A) Preoperative and postoperative pelvic radiographs of a typical case. The images show that the centre of rotation (COR), horizontal distance (HD) and vertical distance (VD) have returned to normal after surgery. (B) Schematic diagram of the two main steps (installation of the PSI and grinding down of the acetabulum) of the new PSI surgical method. (C,D,E,F) Application of the PSI during surgery. 


\section{Crowe IV:}
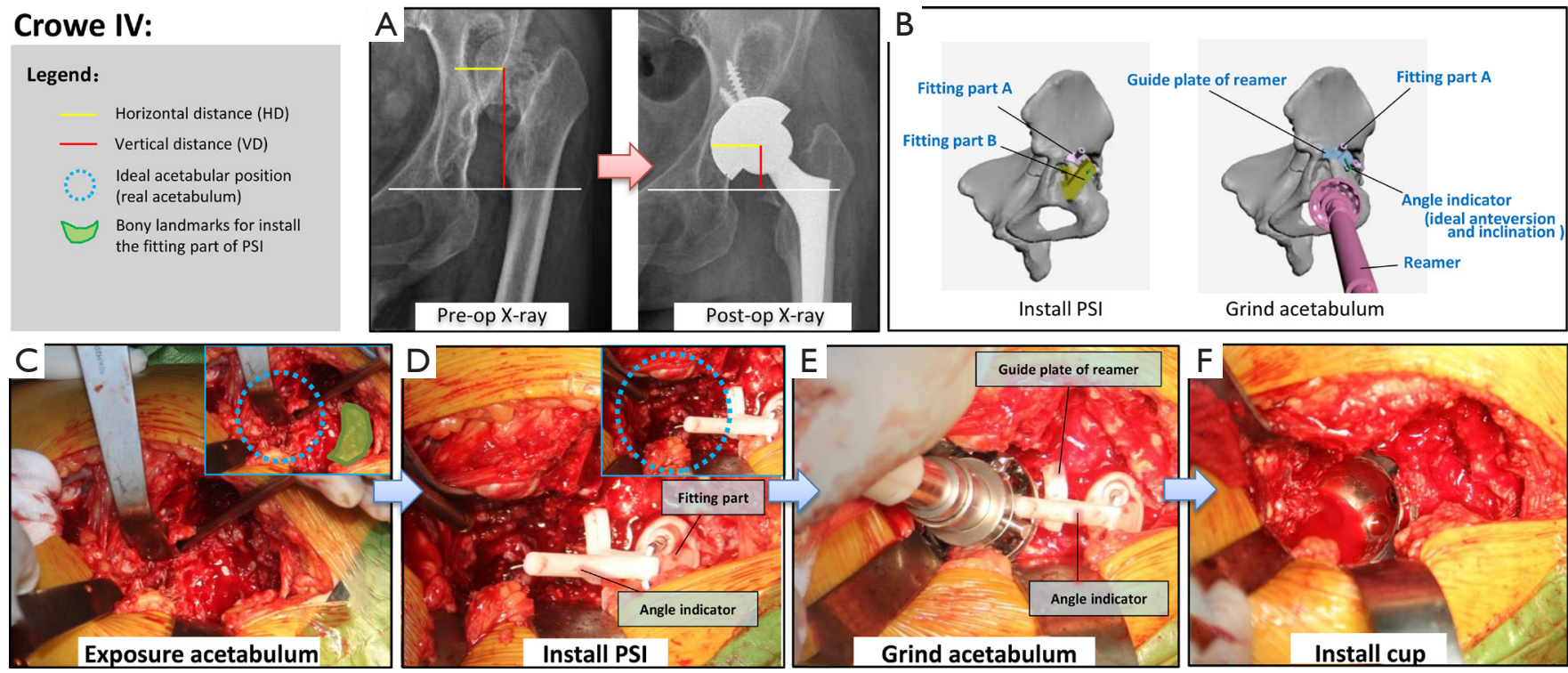

Figure 3 Details of application of the new PSI for patients with Crowe IV developmental dysplasia of the hip (DDH). (A) Preoperative and postoperative pelvic radiographs of a typical case. The centre of rotation (COR), horizontal distance (HD) and vertical distance (VD) have returned to normal after surgery. (B) Schematic diagram of the two main steps (installation of PSI and grinding down of the acetabulum) of the new PSI surgical method. (C,D,E,F) Application of the PSI during surgery.

placement of the cup; the third end, the angle indicator, was designed to guide the orientation of the cup. The final PSI was based on the anatomical structure of the acetabular contour. We used nylon material to construct the PSI and pelvis, using the selective laser sintering (SLS) technique. After strict inspection, only qualified instruments were sterilized and used during surgery. An animated simulation of PSI preoperative design and the surgical process is presented in Videos S1-S3. An example of the design process of the cup and PSI is shown in Video S4.

\section{Surgery}

The surgeons exposed the acetabulum via a traditional hip posterolateral incision. Surgery was then performed by conventional methods in the $\mathrm{CO}$ group. However, there were several differences between the surgical procedures performed in the PSI group and CO group (Table 1, Figures 1-3). In the PSI group, (I) the position of the true acetabulum was determined according to the arc-shaped end of the PSI; (II) if the location of the true acetabulum had been accurately determined, surgeons were able to clean the acetabulum and surrounding area within reasonable limits; (III) surgeons were then able to accurately grind the acetabulum; guided by the PSI, the most optimal orientation of the cup was determined by the angle indicator, and the most optimal size and position of the cup was established by the arc-shaped end; (IV) the surgeons accurately installed the cup, and the most optimal orientation of the cup was again confirmed by the angle indicator. All surgeries were performed with non-cemented prosthetic products obtained from two companies (Zimmer, Inc., Warsaw, IN, USA; DePuy Orthopaedics, Inc., Warsaw, IN, USA).

\section{Variables, outcome measures, data sources, and bias}

Each participant underwent a preoperative radiographic examination [pelvic (Figures 1-3), hip lateral, and full length of both legs] and CT scans, and CT 3D reconstruction within 10 days after surgery. Before surgery, in both the PSI and CO groups, the ideal cup angle, cup position, femoral head size, and femoral head position were designed by computerized simulation (all surgeons were blinded to the preoperative simulation design of the $\mathrm{CO}$ group). The intraoperative conditions of all participants were recorded, including the surgical time for each procedure, intraoperative blood loss, complications, and specific steps.

For the postoperative evaluation, each participant underwent radiographic examination and CT scan between 


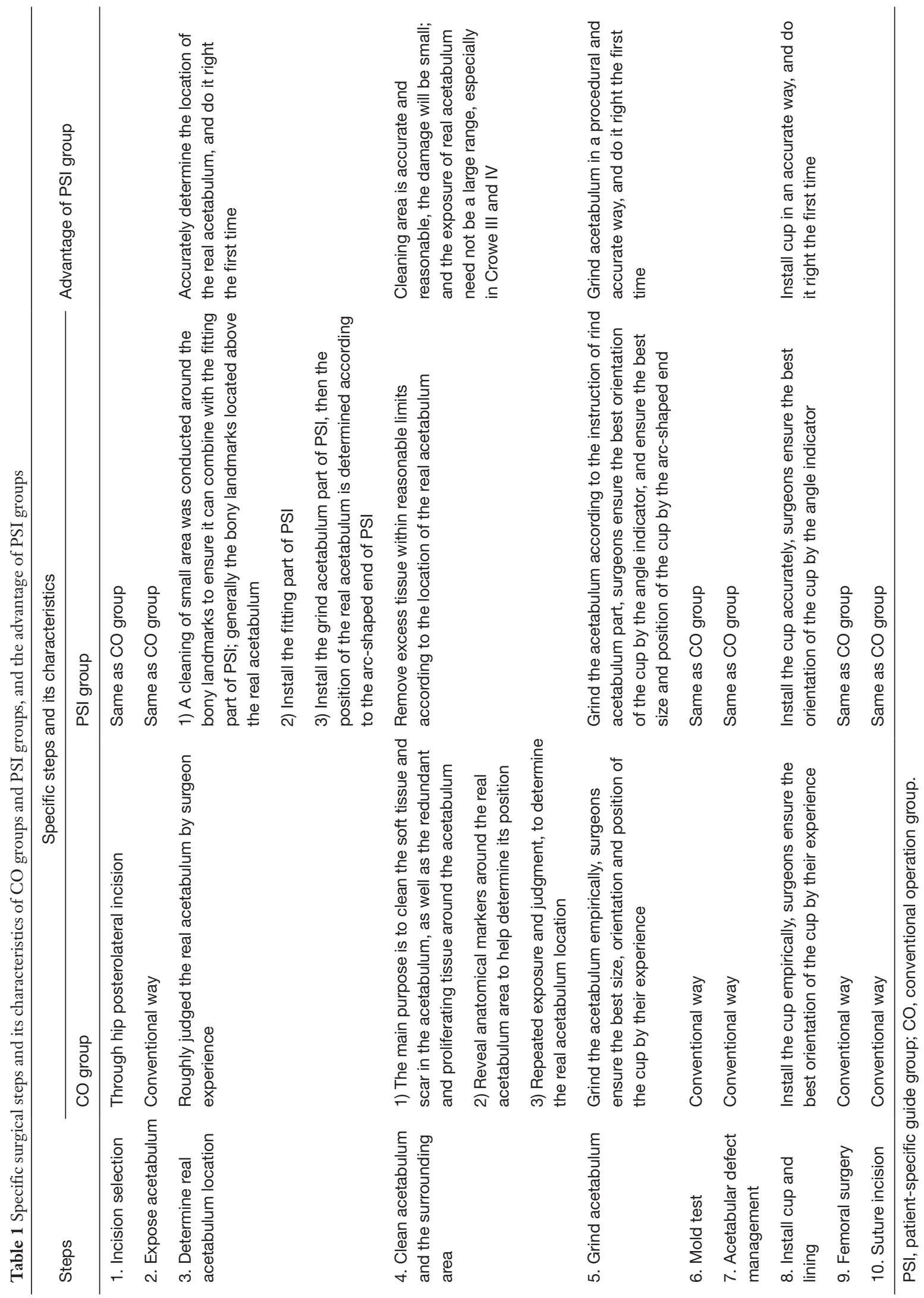


days 3 and 21 after surgery. We recorded the COR as the rotator central position of the femoral head (RCPFH) in the preoperative plan and postoperatively. We then considered the distance between the postoperative RCPFH and preoperative $\mathrm{RCPFH}$ as the ideal rotator centre discrepancy (IRCD). Follow-up was conducted within one year after surgery. For all subgroups, we recorded any related complications, Harris hip scores (HHS), and radiographic results at 3 and 12 months after surgery. After collection of all the data, blinded senior physician teams made their assessments based on the following four aspects:

(I) Ability to carry out preoperative planning of each group: within eight groups, the anteversion, inclination, and PACCs of preoperative computerized simulation plans were paired and compared with the corresponding postoperative measurements.

(II) Accuracy comparison between each subgroup pair: for each Crowe type, the anteversion, inclination, PACCs, qualification rate of the PACC, IRCD, and qualification rate of the IRCD were compared between the PSI and CO groups.

(III) Intraoperative comparison between each subgroup pair: in each Crowe type, the time taken for each step of the surgical procedure, the total surgical time, intraoperative blood loss, and proportion of specific steps in the PSI group were compared with corresponding indexes in the $\mathrm{CO}$ group.

(IV) Function and complications comparison between each subgroup pair: in each Crowe type, the HHS at various time points and complications were compared between the PSI and CO groups.

\section{Demographics, description of the study population}

The baseline data were recorded for all groups prior to surgery, including sex, age, surgical side, and preoperative HHS (28) (Table 2). The age range of the participants was 18-85 years, among both sexes and both surgical sides. There were no significant differences in the number of patients, age, sex, side, and HHS between each pair of subgroups. Female patients outnumbered male patients regardless of the classification.

\section{Patient protocols}

During the trial period, 163 adult patients underwent DDH-THA at the trial centre, among which, 59 patients were excluded (Figure 4). First, 104 participants were categorized based on Crowe's classification (29). Pelvic radiographs were examined by senior physicians, who were blinded to the specific patient grouping during the entire study. All 104 participants were then randomized to either the PSI group (52 patients) or the CO group (52 patients). All participants were further divided into eight subgroups: PSI-Crowe I ( $\mathrm{n}=21)$; PSI-Crowe II $(\mathrm{n}=13)$; PSI-Crowe III $(\mathrm{n}=8)$; PSI-Crowe IV $(\mathrm{n}=10)$; CO-Crowe I $(\mathrm{n}=21)$; COCrowe II ( $\mathrm{n}=14)$; CO-Crowe III $(\mathrm{n}=7)$; and CO-Crowe IV $(\mathrm{n}=10)$. Follow-up was conducted at 3 and 12 months after surgery. The mean follow-up time was $15.12 \pm 2.92$ months, and 102 patients were followed-up twice. One PSI-Crowe II patient who migrated to another country 5 months after surgery completed follow-up at 12 weeks. One COCrowe I patient who withdrew from the study at 10 weeks postoperatively was not followed up.

\section{Randomization}

Participants were randomized to either the PSI group or CO group using random number generators, and all participants were divided into eight subgroups by stratified random sampling based on Crowe classification. Because of the blind design of this study, the senior physicians who classified cases and evaluated results did not know the specific operation procedure or how patients were grouped.

\section{Statistical analysis}

The paired-samples $t$-test was used to analyse the preoperative and corresponding postoperative measures in all groups. The independent samples $t$-test was used to evaluate any differences in surgical time, intraoperative blood loss, postoperative cup positioning, IRCD, PACC discrepancy, and HHS between the PSI group and the CO group among each Crowe type. The chi-square test was used to analyse any differences between the sexes, sides, qualification rates of PACC and IRCD, and proportion of specific steps between the PSI and CO groups among each Crowe type. Statistical analyses were performed using SPSS 20.0 software (SPSS Inc., Chicago, IL, USA). P values less than 0.05 were considered statistically significant.

\section{Results}

The accuracy of cup orientation and positioning showed little difference between the PSI groups and CO groups in patients with Crowe I and Crowe II DDH. However, 


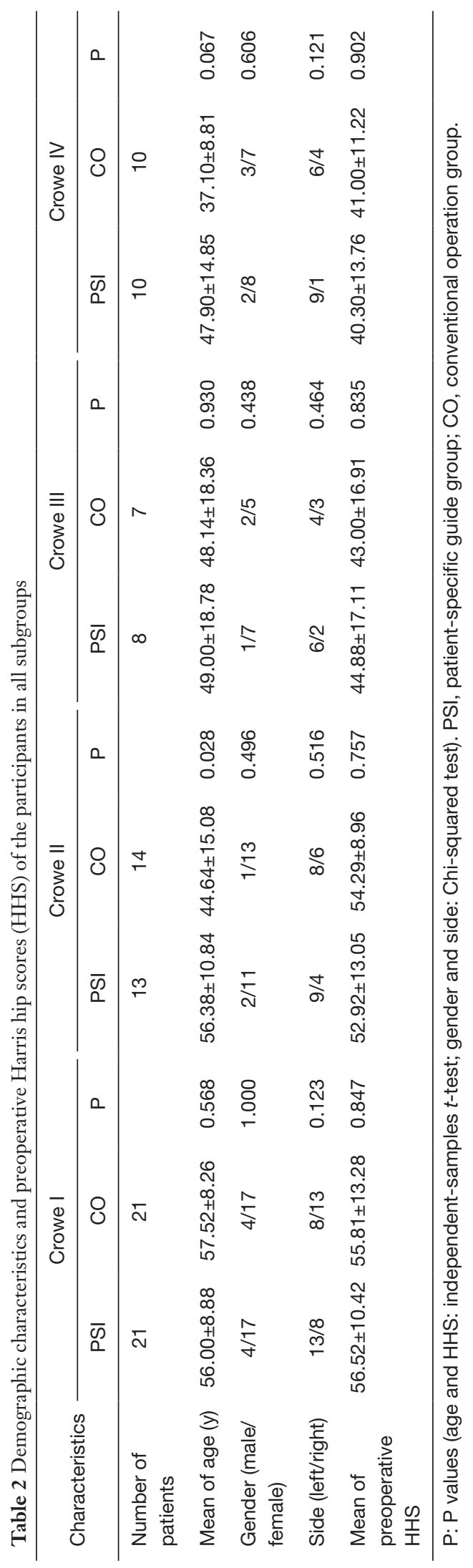

the PSI groups showed much more favourable results than the CO groups in patients with Crowe III and IV DDH. Although there was no significant difference in cup orientation and positioning between the PSI and CO groups in patients with Crowe I and II DDH, significant differences were noted between these two groups in patients with Crowe III and IV DDH.

The ideal values were predicted by the preoperative computerized simulation plan. In the CO-Crowe II group, postoperative anteversion was significantly less than that in the preoperative plan. In the CO-Crowe III group, the postoperative anteversion and postoperative PACC were significantly less than the ideal anteversion and ideal PACC. Furthermore, postoperative inclination was significantly greater than that of the preoperative plan. In the CO-Crowe IV group, postoperative anteversion was significantly less than ideal anteversion, and postoperative inclination was significantly greater than that of the preoperative plan (Table 3).

As shown in Figure 5, in all PSI groups (Crowe I, II, III, or IV), as well as the CO-Crowe I and II groups, the cup orientations were almost always in the preoperative plan zone or Lewinnek safe zone (20), with more favorable results for all PSI groups. However, in the CO-Crowe III and IV groups, several cup orientations were outside of the Lewinnek safe zone, with opposite findings in the PSICrowe III and IV groups. Figure 6 shows that we observed similar outcomes among some groups. In the CO-Crowe III and IV groups, many PACC values were not within the qualified area.

A comparison of the indexes of postoperative measurements between each pair of subgroups indicated that the postoperative anteversion in the CO-Crowe III group was significantly less than that in the PSI-Crowe III group. The postoperative inclination in the CO-Crowe III group was significantly greater than that in the PSI-Crowe III group. The postoperative PACC in the CO-Crowe III group was significantly less than that in the PSI-Crowe III group. The IRCD of the CO-Crowe III group was significantly greater than that of the PSI-Crowe III group. In the PSI-Crowe III group, the qualification rates of the PACC and IRCD were significantly higher than those in the CO-Crowe III group. Postoperative anteversion in the CO-Crowe IV group was significantly less than that in the PSI-Crowe IV group, and postoperative inclination in the CO-Crowe IV group was significantly greater than that in the PSI-Crowe IV group. The IRCD of the CO-Crowe IV group was significantly greater than that of the PSI-Crowe IV group, and the qualification rate of the IRCD in the PSI-Crowe IV group 


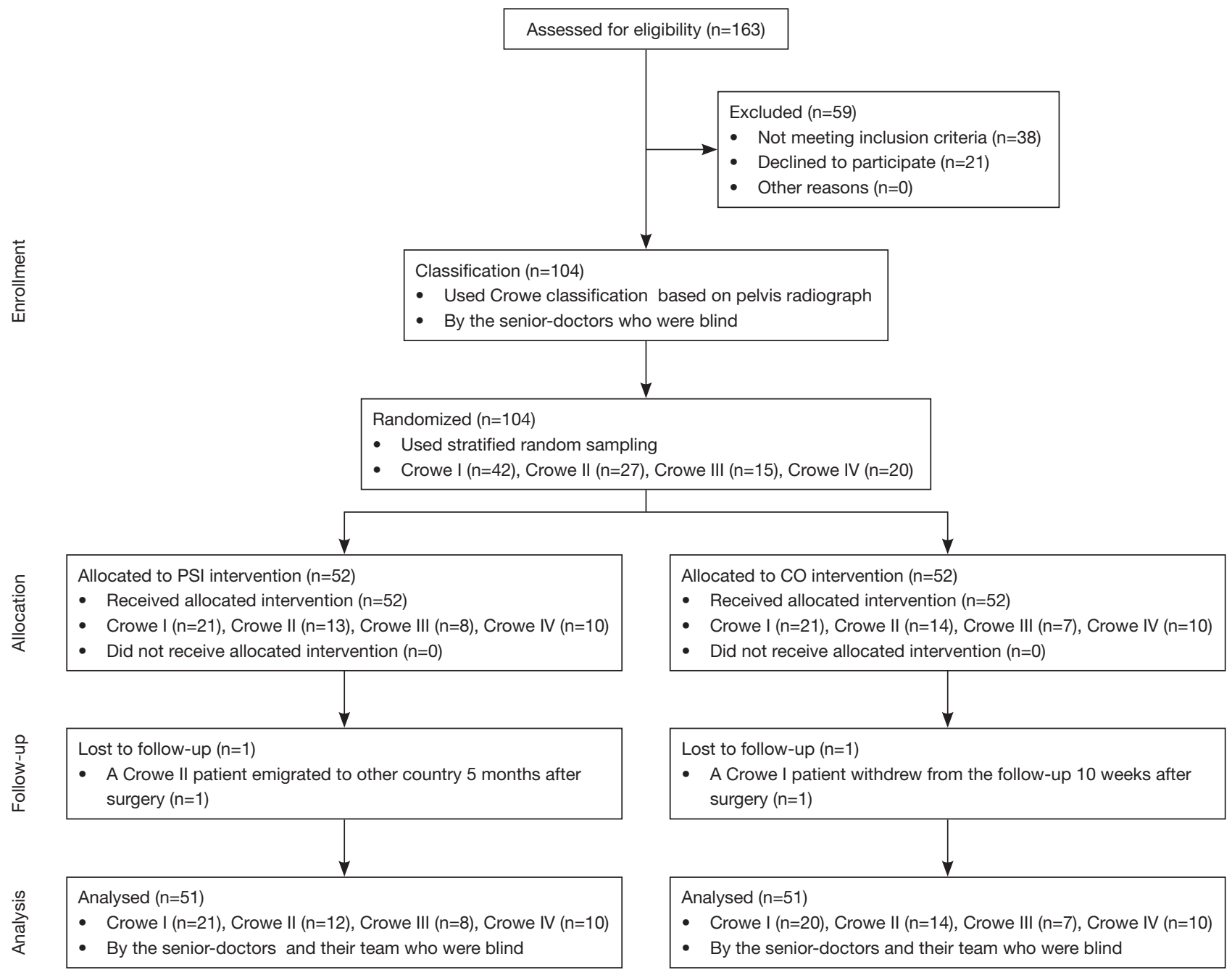

Figure 4 Flow diagram of the allocation of patients in the patient-specific instrumentation (PSI) group and the conventional operation (CO) group. In the blinded study design, the senior doctors and their team, who classified cases and evaluated results, were unaware of the specific operating procedures and grouping of the patients throughout the study.

was significantly better than that in the CO-Crowe IV group (Table 3).

As shown in Figure 5, the PSI and CO groups exhibited roughly the same distribution areas of cup orientation in Crowe I and II DDH patients. However, in patients with Crowe III and IV DDH, the cup orientation distribution areas were significantly different between the PSI and CO groups. The CO-Crowe III and IV groups exhibited less anteversion, and the CO-Crowe III and IV groups exhibited greater inclination. As shown in Figure 6, there was a larger PACC for the PSI-Crowe III group as compared to the CO-Crowe III group, and the PSI-Crowe III and IV groups exhibited a more optimal COR than the CO-Crowe III and IV groups.

In general, more favourable surgical processes were obtained for the PSI groups than the CO groups in patients with Crowe III and IV DDH. In patients with Crowe I DDH, the surgical time required for grinding of the acetabulum in the PSI group was greater than that required for a similar process in the CO group. Similarly, the surgical time required for installation of the cup in the PSI group was greater than that required for the same process in the CO group. In the Crowe III group, the surgical time required for grinding of the acetabulum in the 


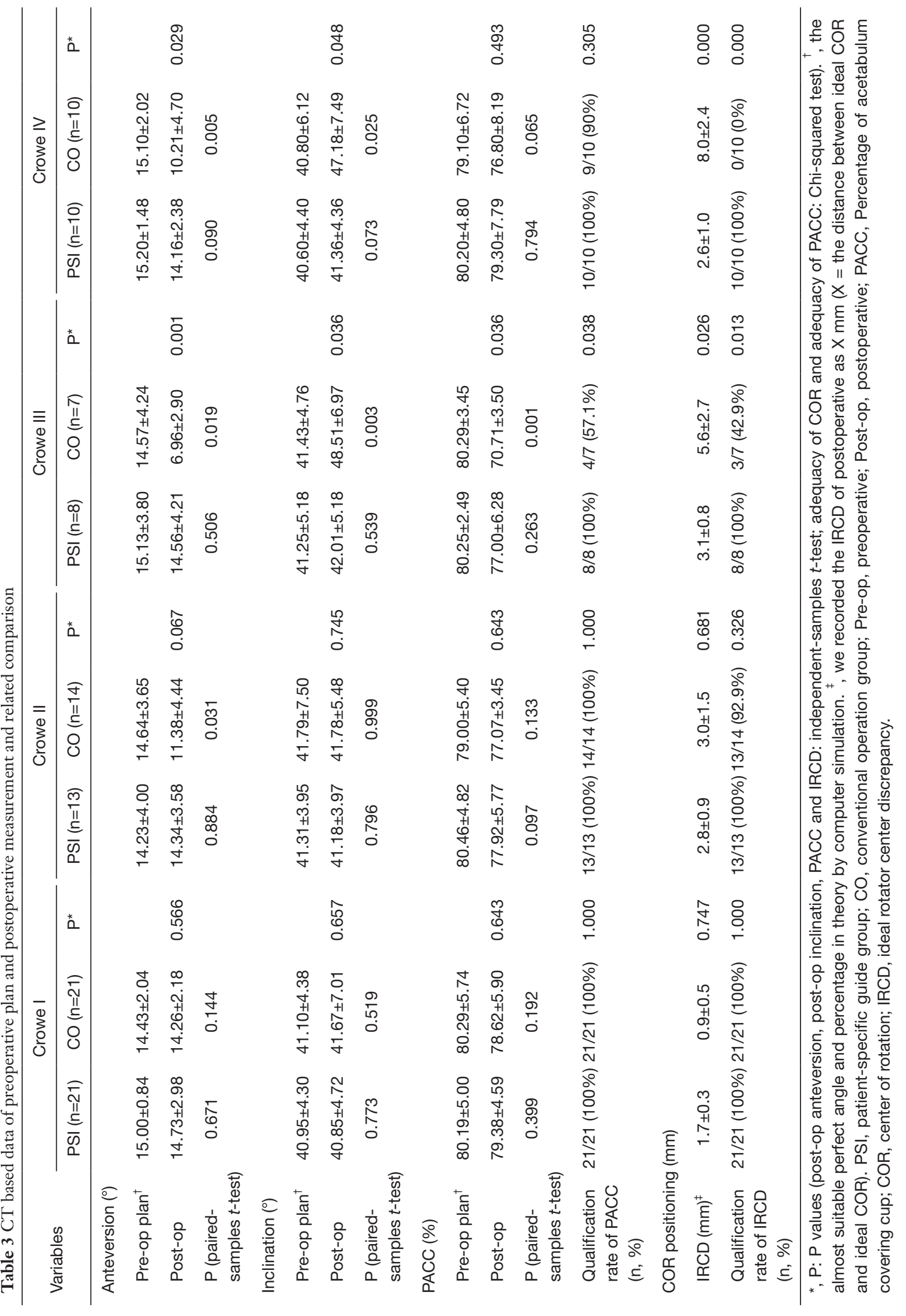


Cup orientation
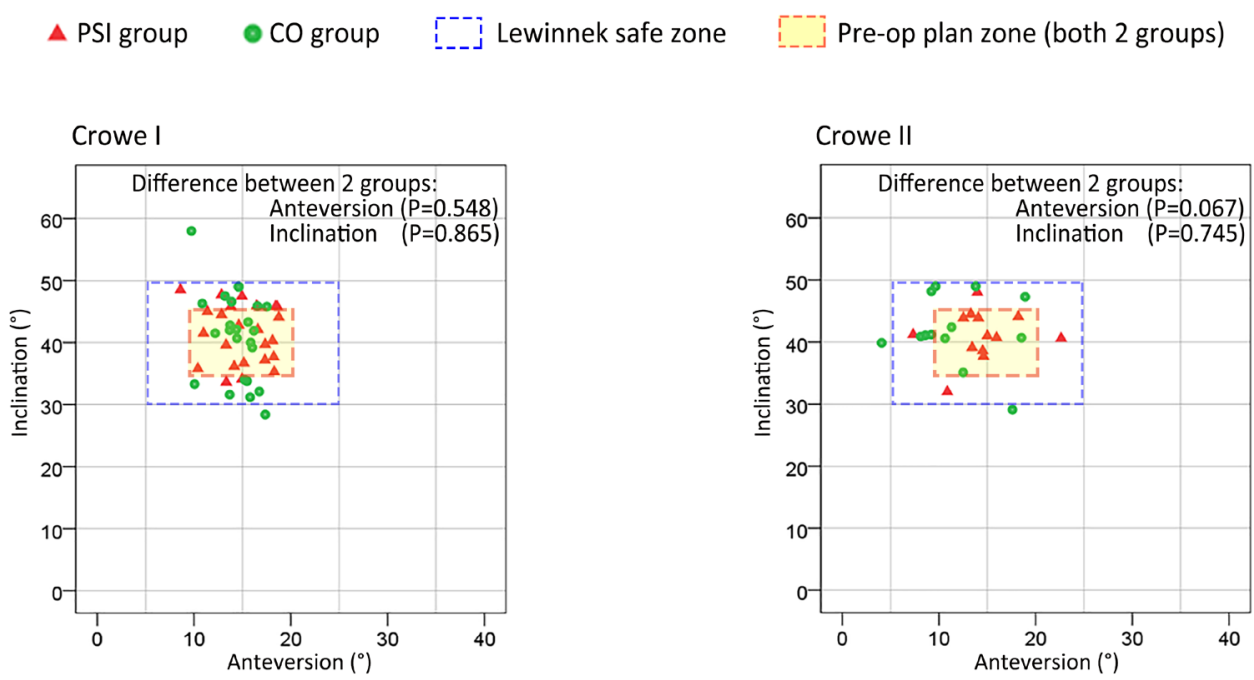

Crowe III

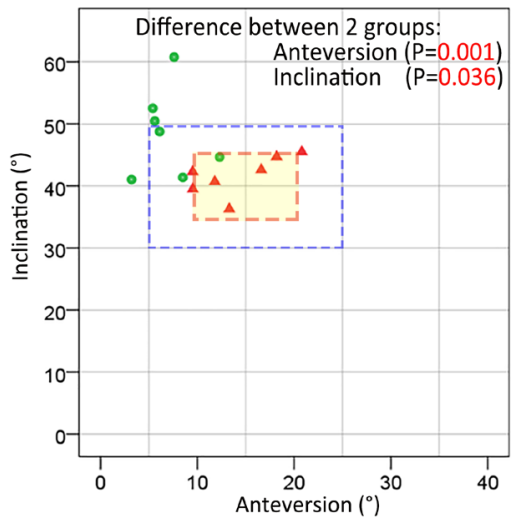

Crowe IV

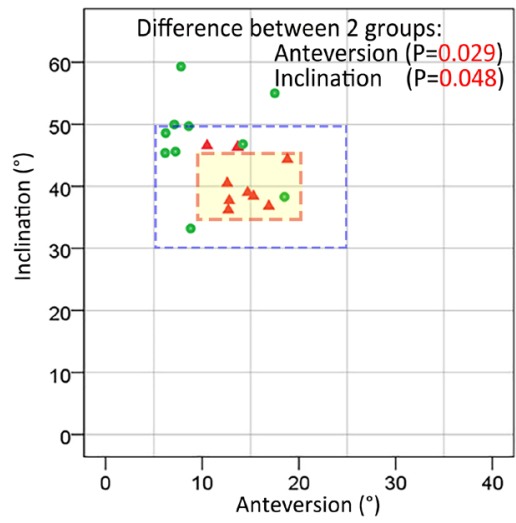

Figure 5 Orientation of the cup prosthesis in all subgroups. The accuracy of cup prosthesis orientation showed little difference between patient-specific instrument (PSI) groups and conventional operation (CO) groups with Crowe I and Crowe II developmental dysplasia of the hip (DDH). However, the accuracy of cup orientation in the PSI groups was much better than that in the CO groups with Crowe III and Crowe IV DDH. The accuracy of the novel PSI in cup prosthesis orientation had a significant advantage over CO in patients with Crowe III and IV DDH, but not in those with Crowe I and II DDH.

PSI group was less than that required for a similar process in the CO group. Similarly, less surgical time was required for installation of the cup in the PSI group than in CO group. The total surgical time required for the PSI group was less than that required for the $\mathrm{CO}$ group. In the Crowe IV group, the surgical time required for grinding of the acetabulum in the PSI group was less than that required for the same process in the CO group. Furthermore, the surgical time required for installation of the cup in the PSI group was less than required for the same process in the $\mathrm{CO}$ group. Intraoperative blood loss in the PSI group was less than that observed in the CO group (Table 4).

As shown in Figures 7 and 8, some outcomes were similar among the various groups. The surgical time was reduced in PSI groups in patients with Crowe III and IV DDH, but surgical time was increased in Crowe I DDH patients. In the PSI group, less intraoperative blood loss was observed in Crowe IV DDH patients. In addition, there was no difference in the proportion of specific steps between PSI groups and CO groups among each Crowe type (Table 4). 


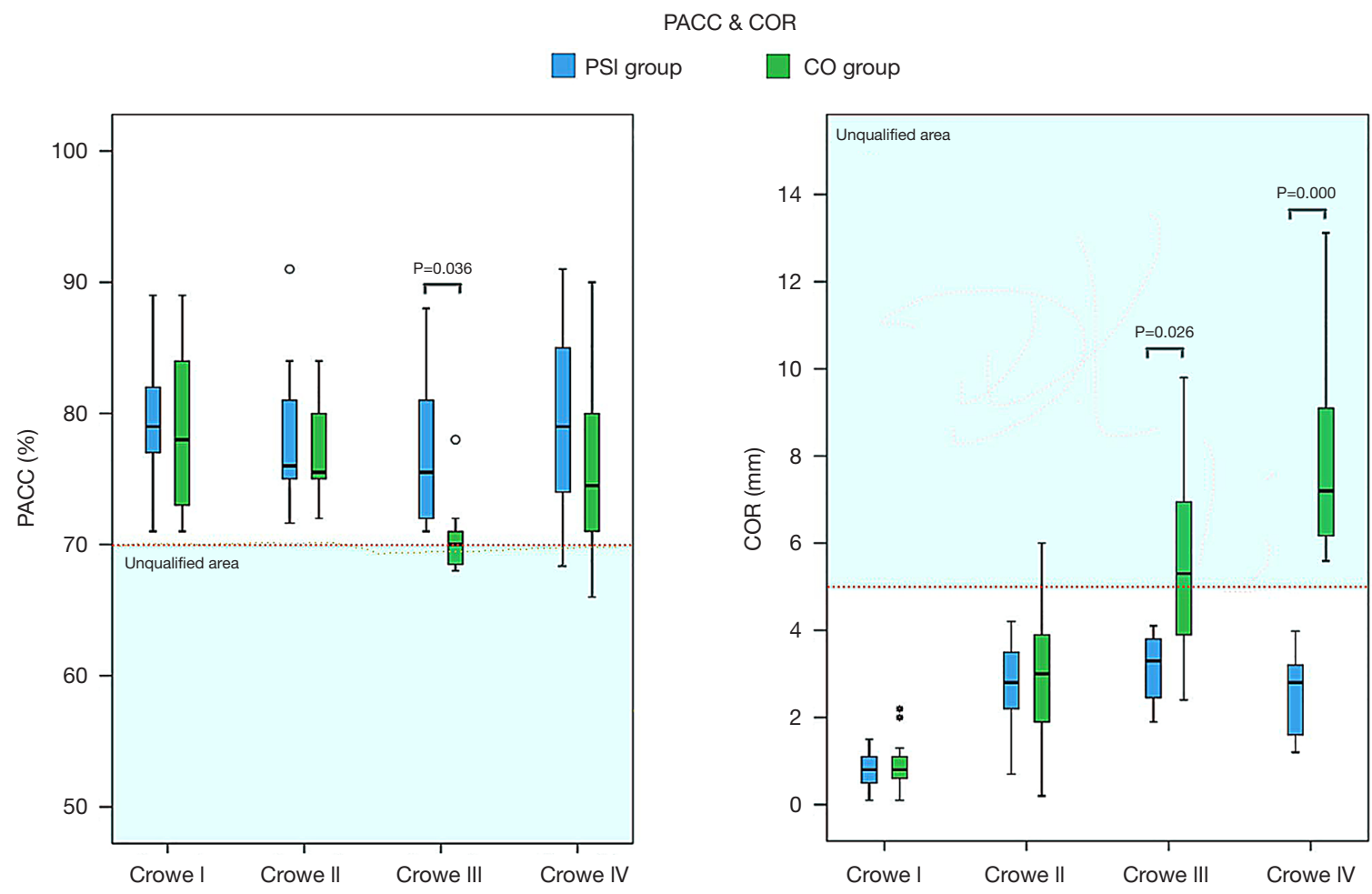

Figure 6 Percentage of acetabular cup coverage (PACC) and centre of rotation (COR) of all subgroups; data are presented as mean (95\% CI). The novel patient-specific instrument (PSI) for qualifying conditions of the PACC showed a significant advantage over the conventional operation (CO) in patients with Crowe III developmental dysplasia of the hip (DDH), but not in those with Crowe I, II and IV DDH. The novel PSI for qualifying conditions of the COR also showed a significant advantage over CO in patients with Crowe III and Crowe IV DDH, but not in those with Crowe I and II DDH. The accuracy of qualifying conditions of the PACC showed no difference between PSI groups and CO groups in patients with Crowe I, II and IV DDH; however, the qualifying conditions of the PACC in PSI groups were much better than those in $\mathrm{CO}$ groups in patients with Crowe III DDH. **, COR of two cases of the CO-Crowe I group.

There were fewer complications in the PSI groups as compared to the CO groups, and the HHS among all subgroups were improved as time progressed. Among patients with Crowe III DDH in the PSI group, more favourable HHS was noted during the third month postoperatively, as compared with the $\mathrm{CO}$ group. In patients with Crowe III and IV DDH, there were better HHS in the PSI groups during the 12th month postoperatively, as compared with the $\mathrm{CO}$ groups.

A total of 102 patients were followed-up twice. One PSI-Crowe II patient, who migrated to another country 5 months after the surgical procedure, completed followup at only 12 weeks after surgery. In addition, one COCrowe I patient, who withdrew from the study 10 weeks after the procedure, was not followed up. One PSI-Crowe III patient developed deep venous thrombosis on day 3 postoperatively, and one CO-Crowe IV patient developed deep venous thrombosis on day 2 postoperatively; they both recovered 3 weeks after surgery. One CO-Crowe IV patient developed transient paralysis of the common peroneal nerve immediately after surgery and recovered 6 months after. One CO-Crowe IV patient developed transient paralysis of the femoral nerve immediately after surgery and recovered 5 months later (Table 5). As shown in Figure 9, the HHS in all subgroups showed improvement as time progressed. In all subgroups, the HHS was improved from baseline to 3 months after surgery and remained reasonably stable from that point to 12 months after surgery. In patients with Crowe III DDH, the HHS at 3 months after surgery in the PSI group was better than that in the CO group, and the HHS at 12 months after surgery in the PSI group was better than that in the CO group. In Crowe IV, the HHS 


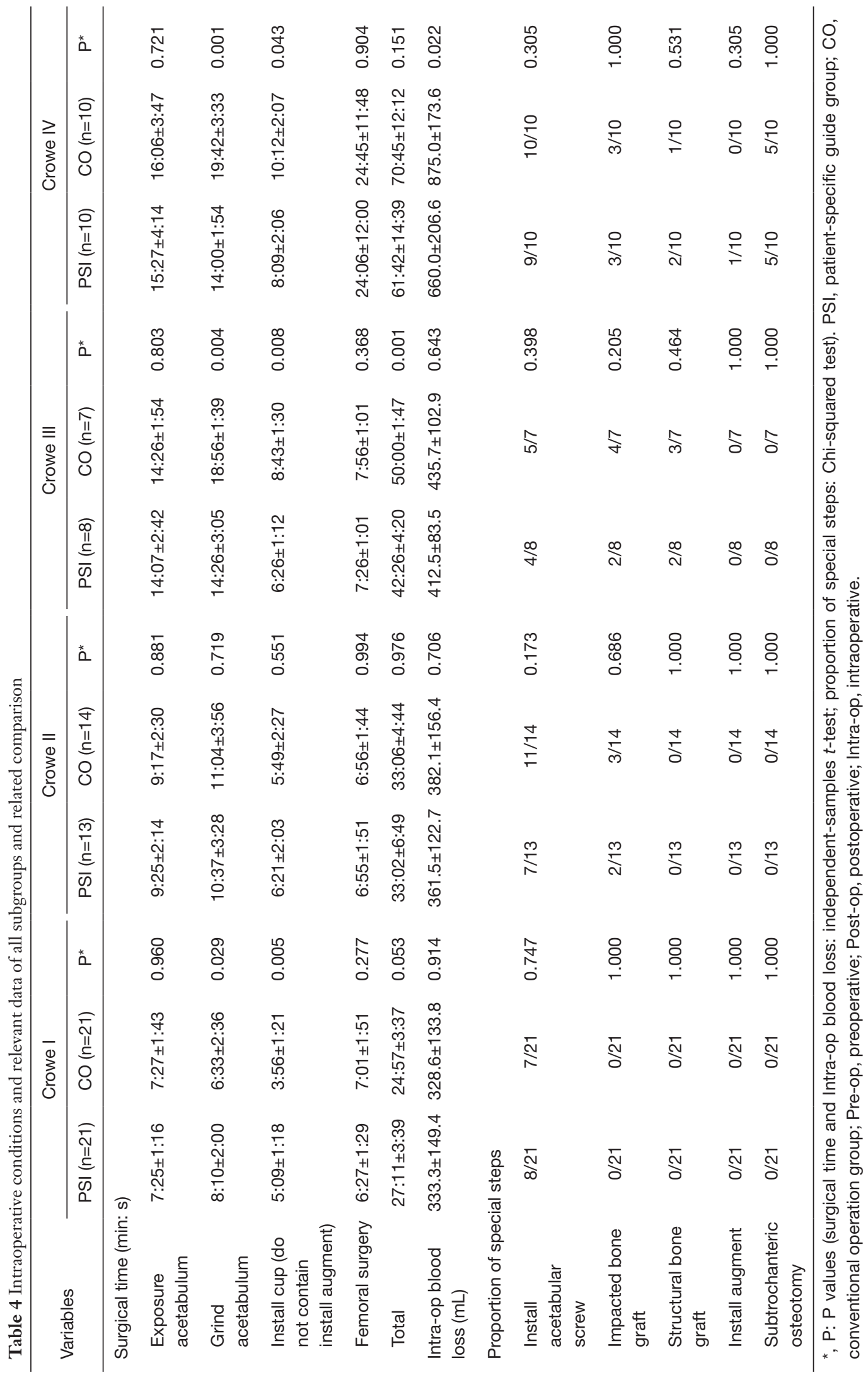


Surgical time

$\square$ PSI group CO group
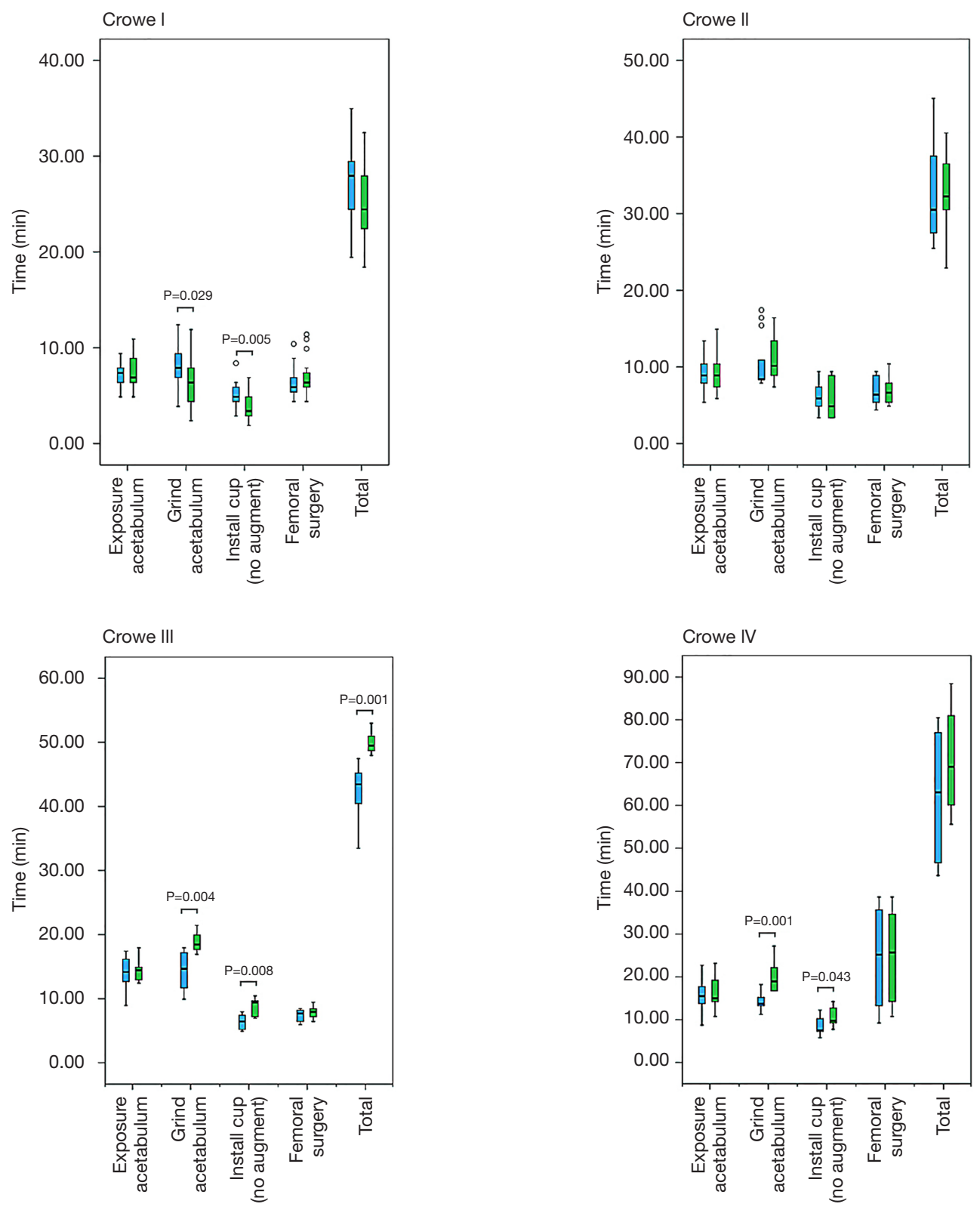

Figure 7 Timing of each step of the surgical procedure and total surgical time of all subgroups; data are presented as mean (95\% CI). In patients with Crowe I developmental dysplasia of the hip (DDH), the surgical time required for grinding of the acetabulum in the patientspecific instrument (PSI) group was greater than that in the conventional operation (CO) group, and the time required to install the cup in the PSI group was greater than that in the CO group. In patients with Crowe III and Crowe IV DDH, a shorter surgical time was required for grinding of the acetabulum and installation of the cup in the PSI groups than in the CO groups. In patients with Crowe III DDH, the total surgical time required for the PSI group was shorter than that required for the CO group. 


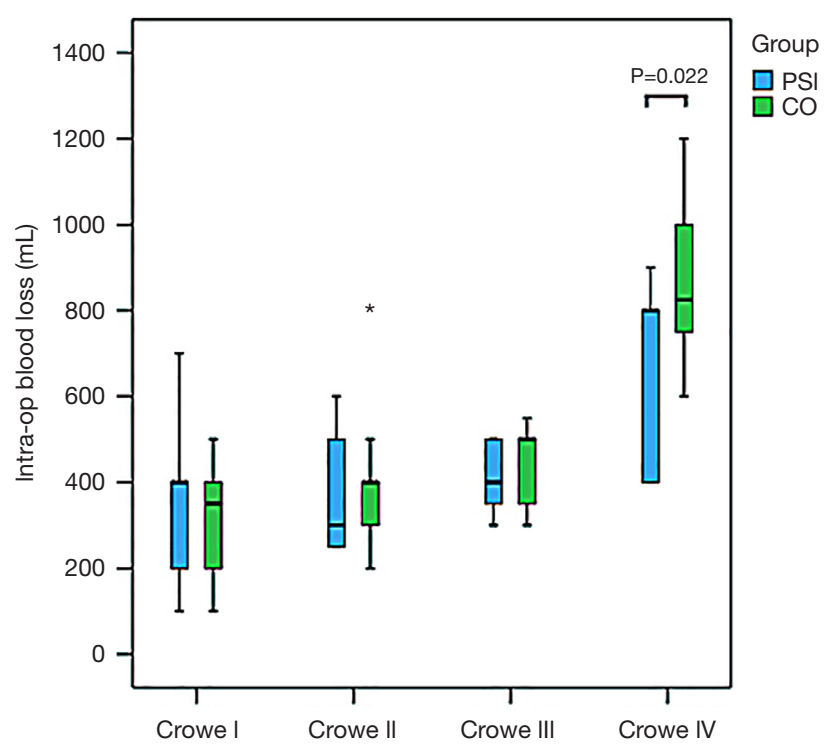

Figure 8 Intraoperative blood loss in all subgroups; data are presented as mean ( $95 \% \mathrm{CI})$. Intraoperative blood loss in the patient-specific instrument (PSI) group was less than that in the conventional operation (CO) group in patients with Crowe IV developmental dysplasia of the hip (DDH). *, blood loss in one case of the CO-Crowe II group.

at 12 months after surgery in the PSI group was better than that in the CO group (Table 5).

\section{Discussion}

At present, construction of an acetabulum with an ideal size and position in adult DDH-THA, and installation of a cup with satisfactory orientation and position, especially for patients classified as Crowe III and IV, remains a challenge $(10,30)$. There is now a general consensus on the type of acetabulum required for reconstruction in DDH patients. However, the location and size of the true acetabulum are difficult to determine because DDH could be the result of several anatomical abnormalities (10). The conventional THA surgical methods rely on manual freehand techniques, which can be inconsistent and unsatisfactory (30-32). Therefore, after more than six years of progressive research and exploration, our team has invented and gradually improved a novel PSI, which has been widely used in our clinical practice. This PSI has the ability to accurately facilitate adult DDH-THA, especially in Crowe III and IV DDH patients. Furthermore, this new PSI can aid in the reconstruction of an ideal acetabulum and installation of a cup in the ideal direction.

This study had a number of limitations. The surgeons in this study were all experienced, and further study is necessary to investigate whether this new PSI can assist surgeons less experienced in performing adult DDHTHA. Nevertheless, we believe this limitation does not alter our conclusions. Generally speaking, adult DDHTHAs are performed by senior surgeons, who still find the operation to be challenging. In most cases, the results obtained from the present study involving senior surgeons are still applicable. Another limitation was the lack of longterm follow-up after surgery. However, we believe this limitation does not compromise our conclusions because this study focused on the improvement of PSI-assisted adult DDH-THA in the perioperative and early postoperative periods. This is likely the first RCT to comprehensively compare PSI-assisted and conventional methods in adult DDH-THA. The positive results obtained by the RCT will provide valuable reference values for further follow-up studies over a longer term.

This trial showed that the accuracy of this PSI is relatively high in adult DDH-THA. This occurred because the preoperative analysis, based on CT data, showed high reliability. Other THA-PSI studies used magnetic resonance imaging (MRI) data, which is easily affected by various soft tissue signals and thus not as reliable as CT data. The preoperative surgical simulation based on CT 3D data was also very accurate. Surgeons were able to repeatedly verify and measure the COR, PACC, anteversion, and inclination, enabling them to reconstruct a perfect virtual acetabulum. Additionally, we used the superolateral rim of the acetabulum as a positioning marker, which is stable, reliable, and accurate. Given the limited soft tissue coverage of the superolateral rim of the acetabulum, it is easy to completely determine the true osseous boundaries.

Some PSI positioning markers are designed for the acetabular fossa (33), which contains a large amount of soft tissue. Because it is very difficult to completely remove all of the soft tissue to reveal the true osseous boundaries, substantial errors may occur (31). Regarding the raw materials and preparation, our new PSI uses SLS technology. In contrast, most other similar studies have used fused deposition modelling (FDM) or stereo lithography appearance (SLA) $(24,33)$. One disadvantage of FDM is the low melting point of its products (19), and their deformation upon high-temperature disinfection. The disadvantage of SLA is the tendency of its products to become brittle after disinfection, and easily break during surgery. 


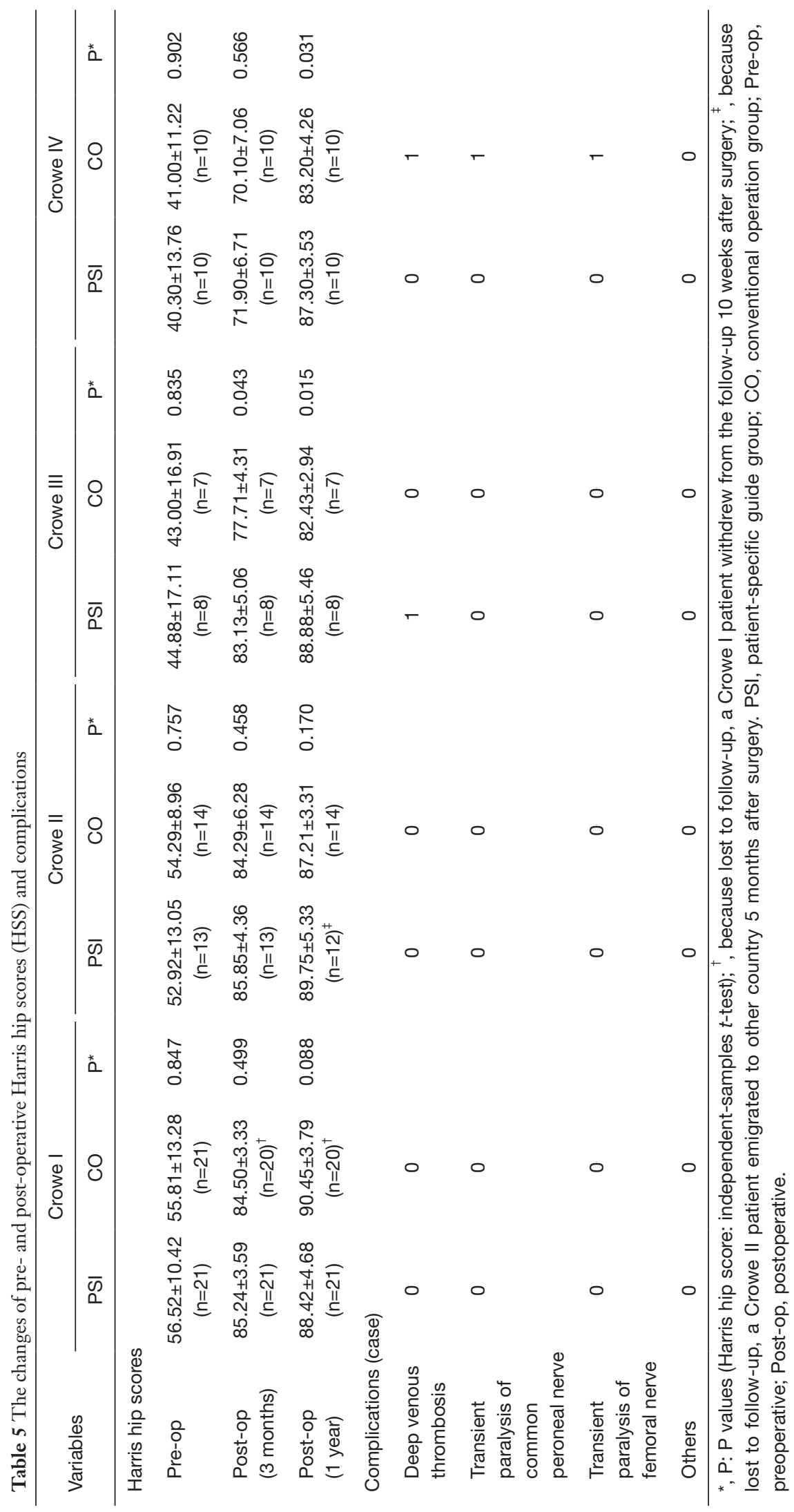


Harris Hip Scores

$\square$ PSI group $\quad \square$ CO group
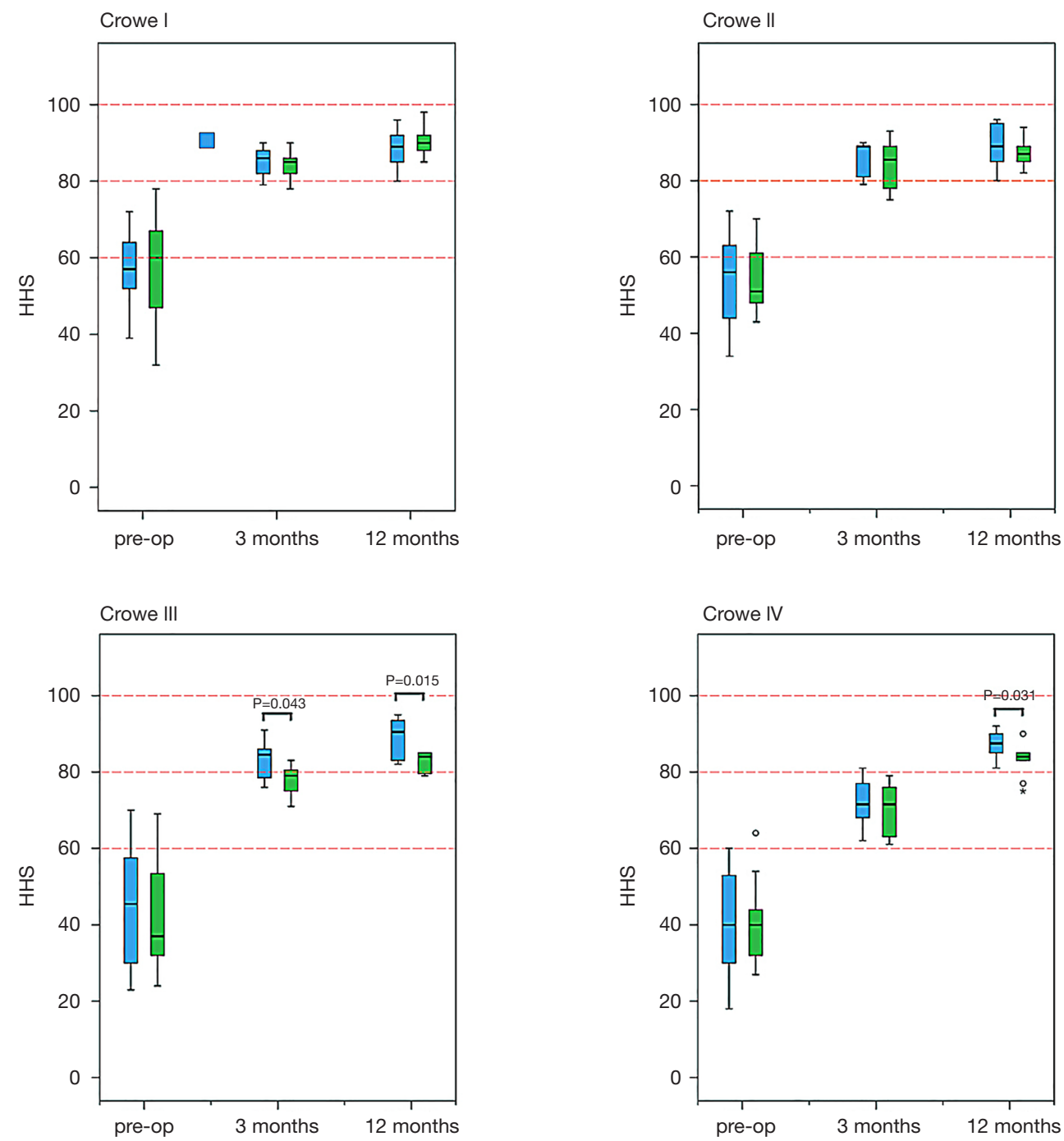

Figure 9 Temporal trends of Harris hip scores (HHS) after surgery in all subgroups; data are presented as mean (95\% CI). The HHS of all subgroups improved as time progressed. In patients with Crowe III and Crowe IV developmental dysplasia of the hip (DDH), the patientspecific instrument (PSI) groups showed better HHS 12 months after surgery than the conventional operation (CO) groups. In patients with Crowe III DDH, the PSI group showed better HHS 3 months after surgery than the CO group.

This trial showed that the accuracy of cup placement, as facilitated by the PSI, had a significant advantage over the CO in patients with Crowe III and IV DDH, but not in those with Crowe I and II DDH. There are numerous possible reasons for this. The conventional surgical method mainly depends on observation of the acetabular anatomical structure to determine the position, size, and orientation of the true acetabulum $(6,7,13,34)$. In patients with Crowe I and II DDH, the acetabular deformity was not the most severe. Senior physicians were able determine the true acetabular position through careful observation of the Harris fossa, transverse ligament, and other structures during surgery, combined with the preoperative radiographs and CT images. In addition, the pelvic size and bone mass of Crowe I and II DDH patients are similar to those of normal patients. Therefore, the processes involved in 
reconstruction of the acetabulum in patients undergoing Crowe I or II DDH-THA are not very different from those in patients without DDH undergoing THA.

With conventional methods, there can be considerable differences in the fake acetabulum prosthesis and the true acetabulum, and the acetabular deformities in Crowe III and IV DDH patients can be very severe. In Crowe III DDH, there is little overlap between the acetabular prosthesis and the true acetabulum. The acetabulum presents a shell-shaped structure with a broad upper part and a narrow lower part. The Harris fossa usually presents as a triangular structure. In patients with Crowe type IV $\mathrm{DDH}$, the acetabulum basically maintains a small, shallow, triangular dysplastic state, and the Harris fossa presents as a mostly flat triangle $(35,36)$. Because of these considerable anatomical variations, determination of the true acetabular region is more challenging. Thus, it is difficult to accurately determine the true COR by conventional surgical methods. In patients with Crowe III and IV DDH, the bones are small, and the shape of the acetabulum is deformed, both of which disqualified the PACC, anteversion, and inclination.

With the new PSI-assisted method, the accuracy of acetabular reconstruction mainly depended on the accuracy of the PSI. Based on our results and analyses, as long as the surgical simulation was performed before surgery, the location of the bone positioning marker and installation of the PSI were accurate, and an appropriate acetabulum could have been reconstructed. Our experience and the results of this trial show that accuracy is much more easily achieved with the PSI method than with conventional methods in Crowe III and IV DDH patients. However, no obvious differences were noted in Crowe I and II DDH patients.

This trial showed that the surgical time required for PSI groups was shorter than that required for $\mathrm{CO}$ groups in patients with Crowe III and IV DDH, but longer in patients with Crowe I DDH. We analyzed the possible reasons as follows. For Crowe III and Crowe IV DDHTHA: (I) The surgeons in the PSI groups participated in preoperative design and computerized surgical simulation, which presented greater advantages over the techniques employed by the surgeons in the CO groups, who only observed radiographs and CT images preoperatively $(37,38)$. (II) In determining the true acetabular location in the PSI groups, surgeons were able to accurately determine the location of the true acetabulum the first time. (III) In the PSI groups, cleaning of the acetabulum and surrounding area was accurate and reasonable, and the damage was minimal. Extensive exposure of the true acetabulum was not necessary. (IV) In the PSI groups, grinding of the acetabulum could have been performed in a systematic and accurate manner that was correct on the first attempt. (V) In the PSI groups, installation of the cup could have been performed accurately on the first attempt. For patients with Crowe I DDH-THA: (I) PSI groups showed no advantages in the steps of cleaning the acetabulum and surrounding area, grinding of the acetabulum, and installation of the cup. This is because the anatomical structure in patients with Crowe I and II DDH is relatively simple. Thus, it is easier for surgeons to make accurate judgments. (II) In the PSI groups, extra steps were required, such as cleaning of the bone positioning marker and installation of the PSI when determining the true acetabular location. Furthermore, extra steps such as alignment of the angle indicator during installation of the cup required extra time.

Intraoperative blood loss in the PSI group was less than that in the CO group with Crowe IV DDH, which occurred because there is considerable deformation of the anatomical structure of the acetabulum and its surrounding tissues in Crowe IV DDH patients. The Crowe IV acetabulum basically maintains a small, shallow triangular dysplastic state, and the Harris fossa presents as a mostly flat triangle $(35,36)$. Thus, with conventional THA for Crowe IV DDH, it is necessary for surgeons to identify anatomical markers around the true acetabular area to determine its position, and clean the soft tissue and scar within the acetabulum, as well as the redundant and proliferating tissue around the acetabulum. Considerable surgical repetition is necessary to determine the true acetabular location. However, in the PSI group, surgeons simply remove excess tissue within reasonable limits, based on the location of the true acetabulum, as determined by the PSI. Thus, cleaning of the surrounding area in the PSI group is both accurate and reasonable, and any damage is minimal in patients with Crowe IV DDH. Therefore, exposure and injury in the CO patient group with Crowe IV DDH was greater than those in the PSI group, thereby leading to increased intraoperative bleeding.

This trial showed that hip function in the PSI groups was more optimal than that in the CO groups of patients with Crowe III and IV DDH, but not in those with Crowe I DDH. This may have occurred because compared with the CO groups, the new surgical methods assisted by the PSI can optimise surgical processes and improve accuracy of placement of the acetabular and cup prosthesis for 
adult Crowe III and IV DDH-THA, but not Crowe I and II DDH-THA. However, based on the data provided in this study and relevant references, for the severe DDH of Crowe III and IV, we also do not believe that such small cup orientation deviation must be the reason for the significant difference in the improvement of hip function one year after surgery. In our opinion, the reason may also be due to shorter operative time or reduced intraoperative tissue damage. In addition, it cannot be ignored that for severe DDH patients, the degree of improvement of hip function after THA caused by cup orientation deviation will be enlarged, compared with that of ordinary primary THA. Therefore, a longer follow-up with a larger sample size and even a multicenter prospective study are indeed needed to further clarify the causes of this phenomenon.

It is worth noting that the PSI group showed better HHS 3 months after surgery as compared to the CO group only in patients with Crowe III DDH, but not in those with Crowe IV DDH. We hypothesized that these phenomena might be related to the considerable surgical trauma that is likely in patients with Crowe IV. Because many patients require sub-trochanteric osteotomy, the soft tissues in almost all cases undergo extension and release during the operation, and postoperative joint stability in patients with Crowe IV DDH is typically worse than that in patients with other types (34). In the PSI and CO groups, these factors had a significant effect on recovery of hip function 3 months after surgery.

\section{Conclusions}

Compared with the conventional method, this new PSI-assisted surgical method improves the accuracy of placement of the acetabular and cup prosthesis, optimises surgical processes, reduces complications, and recovers hip function more quickly after surgery in adults undergoing Crowe III and IV DDH-THA; however, only minimal benefit was obtained with the PSI in patients with Crowe I and II DDH.

\section{Acknowledgments}

We thank the technical support by National Digital Research Institute of Orthopedics of Xiangya Hospital.

Funding: This work was supported by National Nature Science Foundation of China (81974360), National Natural Science Foundation of China (81902308) and Natural Science Foundation of Hunan Province (2020JJ5924).

\section{Footnote}

Reporting Checklist: The authors have completed the CONSORT reporting checklist. Available at http://dx.doi. org/10.21037/atm-20-3488

Data Sharing Statement: Available at http://dx.doi. org/10.21037/atm-20-3488

Peer Review File: Available at http://dx.doi.org/10.21037/ atm-20-3488

Conflicts of Interest: All authors have completed the ICMJE uniform disclosure form (available at http://dx.doi. org/10.21037/atm-20-3488). YL serves as an unpaid Section Editor of Annals of Translational Medicine. The other authors have no conflicts of interest to declare.

Ethical Statement: The authors are accountable for all aspects of the work in ensuring that questions related to the accuracy or integrity of any part of the work are appropriately investigated and resolved. The study was conducted in accordance with the Declaration of Helsinki (as revised in 2013). The study was approved by the medical ethics committee of the Xiangya Hospital of Centre South University (No. 201611080) and has been approved on Clinical Trial Registry (ID: ChiCTR1900024076, http://www.chictr.org.cn/showproj.aspx?proj=39100). All procedures performed in studies were in accordance with the ethical standards of our institutional ethical committee. Informed consent was obtained from all individual participants included in the study.

Open Access Statement: This is an Open Access article distributed in accordance with the Creative Commons Attribution-NonCommercial-NoDerivs 4.0 International License (CC BY-NC-ND 4.0), which permits the noncommercial replication and distribution of the article with the strict proviso that no changes or edits are made and the original work is properly cited (including links to both the formal publication through the relevant DOI and the license). See: https://creativecommons.org/licenses/by-nc-nd/4.0/.

\section{References}

1. Dezateux C, Rosendahl K. Developmental dysplasia of the hip. Lancet 2007;369:1541-52.

2. Wassef AJ, Khlopas A, Sodhi N, et al. Use of an offset head center acetabular shell in difficult primary total hip 
arthroplasties. Ann Transl Med 2019;7:75.

3. Siebenrock KA, Kalbermatten DF, Ganz R. Effect of pelvic tilt on acetabular retroversion: a study of pelves from cadavers. Clin Orthop Relat Res 2003:241-8.

4. Goyal P, Lau A, Naudie DD, et al. Effect of Acetabular Component Positioning on Functional Outcomes in Primary Total Hip Arthroplasty. J Arthroplasty 2017;32:843-8.

5. Bjarnason JA, Reikeras O. Changes of center of rotation and femoral offset in total hip arthroplasty. Ann Transl Med 2015;3:355.

6. Greber EM, Pelt CE, Gililland JM, et al. Challenges in Total Hip Arthroplasty in the Setting of Developmental Dysplasia of the Hip. J Arthroplasty 2017;32:S38-S44.

7. Xu J, Xu C, Mao Y, et al. Posterosuperior Placement of a Standard-Sized Cup at the True Acetabulum in Acetabular Reconstruction of Developmental Dysplasia of the Hip With High Dislocation. J Arthroplasty 2016;31:1233-9.

8. Dastane M, Dorr LD, Tarwala R, et al. Hip offset in total hip arthroplasty: quantitative measurement with navigation. Clin Orthop Relat Res 2011;469:429-36.

9. Lewinnek GE, Lewis JL, Tarr R, et al. Dislocations after total hip-replacement arthroplasties. J Bone Joint Surg Am 1978;60:217-20.

10. Baki ME, Timurkaynak A, Aydın H, et al. Metal-on-metal dysplasia cup total hip arthroplasty for hip osteoarthritis secondary to developmental dysplasia of the hip. Eklem Hastalik Cerrahisi 2014;25:154-7.

11. Mainard D, Barbier O, Knafo Y, et al. Accuracy and reproducibility of preoperative three-dimensional planning for total hip arthroplasty using biplanar low-dose radiographs: A pilot study. Orthop Traumatol Surg Res 2017;103:531-6.

12. Lei $\mathrm{P}, \mathrm{Hu} \mathrm{Y}$, Cai $\mathrm{P}$, et al. Greater trochanter osteotomy with cementless THA for Crowe type IV DDH. Orthopedics 2013;36:e601-5.

13. Zhang H, Zhou J, Guan J, et al. How to restore rotation center in total hip arthroplasty for developmental dysplasia of the hip by recognizing the pathomorphology of acetabulum and Harris fossa. J Orthop Surg Res 2019;14:339.

14. Inaba Y, Kobayashi N, Ike H, et al. The current status and future prospects of computer-assisted hip surgery. J Orthop Sci 2016;21:107-15.

15. Inoue D, Kabata T, Maeda T, et al. Value of computed tomography-based three-dimensional surgical preoperative planning software in total hip arthroplasty with developmental dysplasia of the hip. J Orthop Sci
2015;20:340-6.

16. Paprosky WG, Muir JM. Intellijoint HIP®: a 3D minioptical navigation tool for improving intraoperative accuracy during total hip arthroplasty. Med Devices (Auckl) 2016;9:401-8.

17. Gurgel HM, Croci AT, Cabrita HA, et al. Acetabular component positioning in total hip arthroplasty with and without a computer-assisted system: a prospective, randomized and controlled study. J Arthroplasty 2014;29:167-71.

18. Parratte S, Ollivier M, Lunebourg A, et al. No Benefit After THA Performed With Computer-assisted Cup Placement: 10-year Results of a Randomized Controlled Study. Clin Orthop Relat Res 2016;474:2085-93.

19. Kollamaram G, Croker DM, Walker GM, et al. Low temperature fused deposition modeling (FDM) $3 \mathrm{D}$ printing of thermolabile drugs. Int $\mathrm{J}$ Pharm 2018;545:144-52.

20. Ogawa H, Hasegawa S, Tsukada S, et al. A Pilot Study of Augmented Reality Technology Applied to the Acetabular Cup Placement During Total Hip Arthroplasty. J Arthroplasty 2018;33:1833-7.

21. Boudissa M, Courvoisier A, Chabanas M, et al. Computer assisted surgery in preoperative planning of acetabular fracture surgery: state of the art. Expert Rev Med Devices 2018;15:81-9.

22. Xu J, Li D, Ma RF, et al. Application of Rapid Prototyping Pelvic Model for Patients with DDH to Facilitate Arthroplasty Planning: A Pilot Study. J Arthroplasty 2015;30:1963-70.

23. Inoue D, Kabata T, Kimura H, et al. A prospective clinical trial to assess the accuracy of an MRI-based patient-specific acetabular instrument guide in total hip arthroplasty. Eur J Orthop Surg Traumatol 2019;29:65-71.

24. Small T, Krebs V, Molloy R, et al. Comparison of acetabular shell position using patient specific instruments vs. standard surgical instruments: a randomized clinical trial. J Arthroplasty 2014;29:1030-7.

25. Zhao JX, Su XY, Zhao Z, et al. Radiographic assessment of the cup orientation after total hip arthroplasty: a literature review. Ann Transl Med 2020;8:130.

26. Yang Y, Zuo J, Liu T, et al. Morphological Analysis of True Acetabulum in Hip Dysplasia (Crowe Classes I-IV) Via 3-D Implantation Simulation. J Bone Joint Surg Am 2017;99:e92.

27. Ueno T, Kabata T, Kajino Y, et al. Three-Dimensional Host Bone Coverage Required in Total Hip Arthroplasty for Developmental Dysplasia of the Hip 
and Its Relationship With 2-Dimensional Coverage. J Arthroplasty 2019;34:93-101.

28. Harris WH. Traumatic arthritis of the hip after dislocation and acetabular fractures: treatment by mold arthroplasty. An end-result study using a new method of result evaluation. J Bone Joint Surg Am 1969;51:737-55.

29. Crowe JF, Mani VJ, Ranawat CS. Total hip replacement in congenital dislocation and dysplasia of the hip. J Bone Joint Surg Am 1979;61:15-23.

30. Hartofilakidis G, Karachalios T. Total hip arthroplasty for congenital hip disease. J Bone Joint Surg Am 2004;86:242-50.

31. Conn KS, Clarke MT, Hallett JP. A simple guide to determine the magnification of radiographs and to improve the accuracy of preoperative templating. J Bone Joint Surg Br 2002;84:269-72.

32. Grammatopoulos G, Thomas GE, Pandit H, et al. The effect of orientation of the acetabular component on outcome following total hip arthroplasty with small diameter hard-on-soft bearings. Bone Joint J 2015;97B:164-72.

33. Zhang YZ, Chen B, Lu S, et al. Preliminary application of computer-assisted patient-specific acetabular navigational template for total hip arthroplasty in adult

Cite this article as: Wang C, Li Y, Hu Y, Liu H, Wang L, Xie J, Xiao H, Su S, Gao F, Zhong D. Patient-specific total hip arthroplasty is superior to conventional methods for Crowe III and IV adult developmental hip dysplasia: a randomized controlled trial. Ann Transl Med 2021;9(3):212. doi: 10.21037/ atm-20-3488 single development dysplasia of the hip. Int J Med Robot 2011;7:469-74.

34. Hitz OF, Flecher X, Parratte S, et al. Minimum 10-Year Outcome of One-Stage Total Hip Arthroplasty Without Subtrochanteric Osteotomy Using a Cementless Custom Stem for Crowe III and IV Hip Dislocation. J Arthroplasty 2018;33:2197-202.

35. Liu T, Wang S, Huang G, et al. Treatment of Crowe IV developmental dysplasia of the hip with cementless total hip arthroplasty and shortening subtrochanteric osteotomy. J Int Med Res 2019;47:3223-33.

36. Macheras GA, Koutsostathis SD, Lepetsos P, et al. THA following deformities due to congenital dislocation of the hip joint. Hip Int 2014;24 Suppl 10:S29-32.

37. Tserovski S, Georgieva S, Simeonov R, et al. Advantages and disadvantages of $3 \mathrm{D}$ printing for pre-operative planning of revision hip surgery. J Surg Case Rep 2019;2019:rjz214.

38. Yi LH, Li R, Zhu ZY, et al. Anatomical study based on 3D-CT image reconstruction of the hip rotation center and femoral offset in a Chinese population: preoperative implications in total hip arthroplasty. Surg Radiol Anat 2019;41:117-24. 